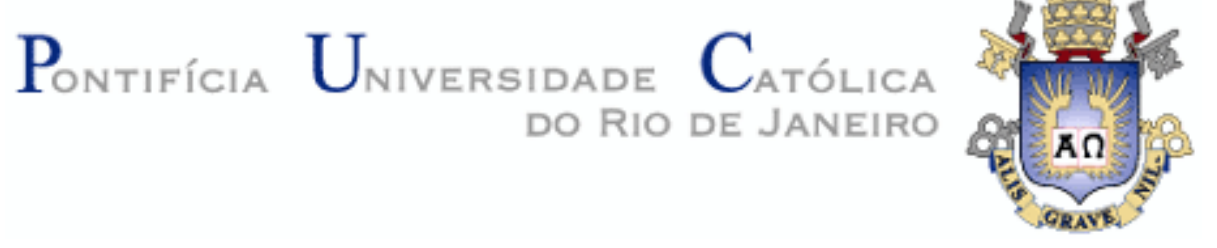

Carl Martin Wehmer

\begin{abstract}
O papel da metrologia e de suas funções correlatas na livre circulação de produtos: considerações sobre a revisão do marco regulatório europeu
\end{abstract}

Dissertação de Mestrado

Dissertação apresentada ao Programa de Pós-graduação em Metrologia da PUC-Rio como requisito parcial para obtenção do título de mestre em Metrologia. Área de Concentração: Metrologia para Qualidade e Inovação.

Orientador: Maurício Nogueira Frota, Ph.D.

Rio de Janeiro, novembro de 2009 


\title{
O papel da metrologia e de suas funções correlatas na livre circulação de produtos: considerações sobre a revisão do marco regulatório europeu
}

\begin{abstract}
Dissertação apresentada ao Programa de Pós-graduação em Metrologia da PUC-Rio como requisito parcial para obtenção do título de mestre em Metrologia. Área de Concentração: Metrologia para Qualidade e Inovação. Aprovada pela Comissão Examinadora abaixo assinada.
\end{abstract}

Prof. Maurício Nogueira Frota, Ph.D. Programa de Pós-Graduação em Metrologia (PósMQI) Pontifícia Universidade Católica do Rio de Janeiro Orientador Hartmut Apel
Physikalisch-Technische Bundesanstalt (PTB)
Co-orientador

Prof. Paul De Bièvre, Ph.D. Diretor do IRMM e Membro do CCQM/BIPM

Prof. Reinaldo Castro Souza, Ph.D. Departamento de Engenharia Elétrica PUC-Rio

Prof ${ }^{a}$. Marina De Almeida Rego Figueira de Mello, Ph.D.

Departamento de Economia PUC-Rio

Prof. José Eugenio Leal Coordenador Setorial do Centro Técnico Científico PUC-Rio 
Todos os direitos reservados. É proibida a reprodução total ou parcial do trabalho sem autorização da universidade, do autor e do orientador.

\section{Carl Martin Wehmer}

Graduado em Administração de Empresas (2000) pela Universidade de Ciências Aplicadas de Regensburg (Alemanha) e Bachelor em Comércio Exterior pela École Supérieure de Commerce de La Rochelle (França). Experiência profissional em comércio exterior e no gerenciamento de projetos.

Ficha Catalográfica

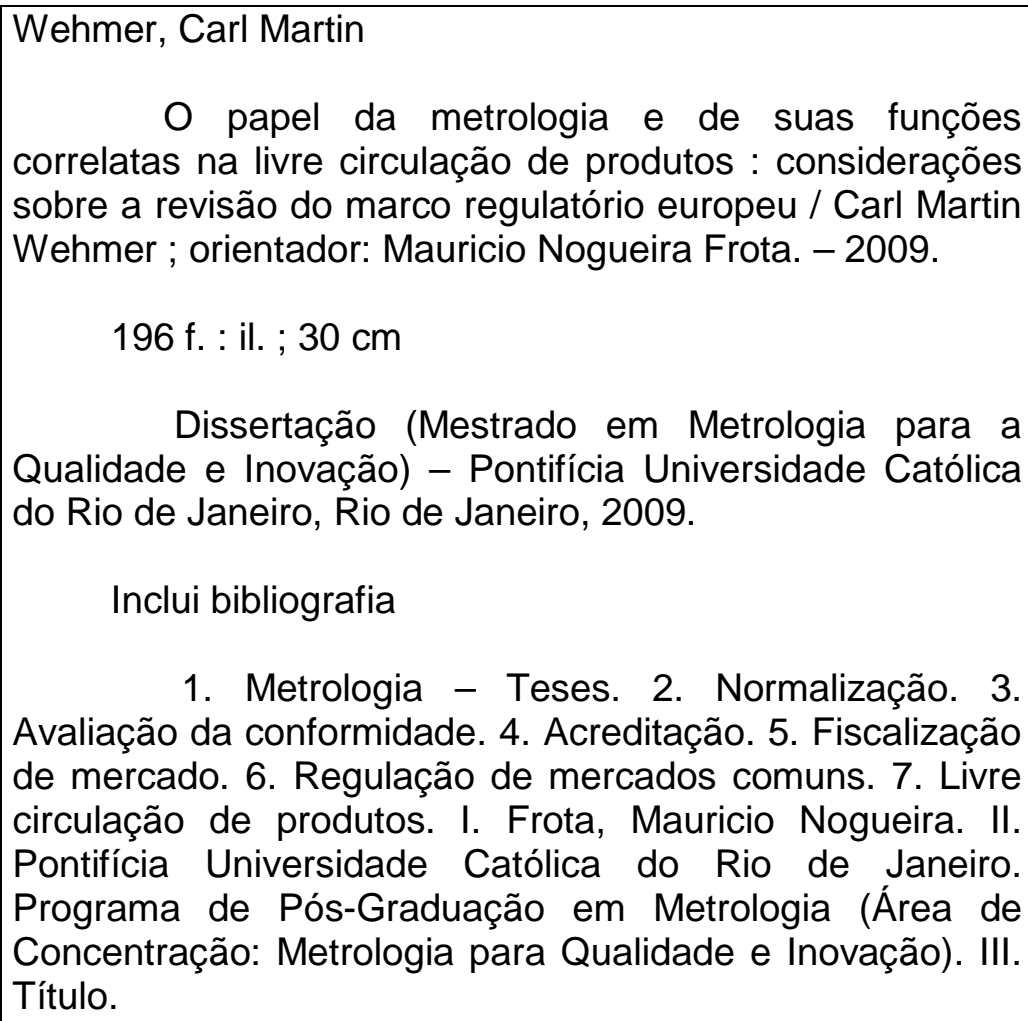

O papel da metrologia e de suas funções correlatas na livre circulação de produtos : considerações sobre a revisão do marco regulatório europeu / Carl Martin Wehmer ; orientador: Mauricio Nogueira Frota. - 2009.

196 f. : il. ; $30 \mathrm{~cm}$

Dissertação (Mestrado em Metrologia para a Qualidade e Inovação) - Pontifícia Universidade Católica do Rio de Janeiro, Rio de Janeiro, 2009.

Inclui bibliografia

1. Metrologia - Teses. 2. Normalização. 3. Avaliação da conformidade. 4. Acreditação. 5. Fiscalização de mercado. 6. Regulação de mercados comuns. 7. Livre circulação de produtos. I. Frota, Mauricio Nogueira. II. Pontifícia Universidade Católica do Rio de Janeiro. Programa de Pós-Graduação em Metrologia (Área de Concentração: Metrologia para Qualidade e Inovação). III. Título. 


\section{Agradecimentos}

Ao meu orientador Professor Maurício Nogueira Frota (coordenador do Programa de Pós-Graduação em Metrologia da PUC-Rio), pelo amplo apoio e dedicada orientação presente em todos os momentos de desenvolvimento da presente dissertação de mestrado. Em particular pela sua visão crítica e abrangente sobre o tema e pelo seu explícito estímulo ao meu desenvolvimento profissional.

Ao co-orientador Hartmut Apel, profissional da metrologia do instituto alemão de metrologia (Physikalisch-Technische Bundesanstalt, PTB), pelas reflexões críticas sobre este complexo tema relacionado à regulação européia para a livre circulação de produtos.

Ao Ministério do Desenvolvimento, Indústria e Comércio Exterior (MDIC) (por intermédio do INMETRO) e à União Européia, pela oportunidade de participar do projeto de Cooperação Técnica Redes de Centros Tecnológicos e Apoio às PME Brasileiras, referência BRA/B7-311/2000/0005. Em particular pela oportunidade de participar do curso oferecido no Brasil sobre o tema objeto da pesquisa e pela oportunidade de participar da missão técnica à Europa que visitou 33 organizações técnicas atuantes em funções da infraestrutura para a qualidade de seis países.

Aos international experts em metrologia, normalização, avaliação da conformidade, acreditação, fiscalização e regulação que aceitaram participar da pesquisa realizada e, demonstrando grande conhecimento sobre o tema, responderam o questionário da pesquisa prestando relevantes informações ao desenvolvimento do trabalho.

Às 33 organizações européias visitadas (todas elas identificadas no Capítulo 4, pelo acesso a relevantes informações e documentos sobre a legislação européia relacionada aos aspectos técnicos, administrativos e regulatórios que fundamentaram o desenvolvimento do trabalho.

À Coordenação de Aperfeiçoamento Profissional de Nível Superior (CAPES), pela bolsa concedida que viabilizou a participação do autor no Programa de Mestrado em Metrologia da PUC-Rio. 
À PUC-Rio, nas pessoas de seus professores e funcionários, pelo ambiente acadêmico-científico favorável ao desenvolvimento deste trabalho. Em particular ao Professor Rui Marques Pitanga, pela leitura dos capítulos centrais da dissertação e a Jaime Mamani Ticona, pelo apoio na formatação do trabalho.

Aos pesquisadores que aceitaram participar da comissão examinadora, dedicando seu tempo na leitura e análise do trabalho. Suas sugestões aqui incorporadas certamente agregaram valor ao trabalho.

À minha família, em particular à minha esposa Pilar, por todo carinho, incentivo e apoio na realização do mestrado no PósMQI/PUC-Rio.

$\mathrm{E}$, também, a todos aqueles que direta ou indiretamente colaboraram para o desenvolvimento do presente trabalho. 


\section{Resumo}

Wehmer, Carl Martin; Frota, Mauricio Nogueira. O papel da metrologia e de suas funções correlatas na livre circulação de produtos: considerações sobre a revisão do marco regulatório europeu. Rio de Janeiro, 2009. 196 p. Dissertação de Mestrado - Departamento de Metrologia (Área de Concentração: Metrologia para Qualidade e Inovação). Pontifícia Universidade Católica do Rio de Janeiro.

A presente dissertação de mestrado tem como objetivo analisar, à luz das funções da infraestrutura tecnológica, as proposições do novo marco regulatório europeu para a livre circulação de produtos no mercado comum. Mais especificamente responder à questão formulada pela pesquisa: conseguirá esse novo marco regulatório equacionar e mitigar as deficiências do modelo anterior? Tendo em vista a atualidade do tema, o trabalho discute a importância da metrologia, da normalização, da acreditação e da avaliação da conformidade na eficiência da fiscalização do mercado em nível global e a relevância técnica e econômica que resultam desses processos. No campo institucional, a motivação pelo tema se deu pelo compromisso do Programa de Pós-Graduação em Metrologia de contribuir para o avanço do conhecimento sobre a regulação de mercados e de explorar o tema como oportunidade de fortalecer a ponte em temas da metrologia com a cidadania. No que concerne a metodologia, a pesquisa desenvolveu-se de forma estruturada segundo as seguintes fases complementares: (i) pesquisa bibliográfica e documental dos textos legais e regulamentos europeus aplicáveis; (ii) pesquisa de campo (iii) construção e aplicação do instrumento da pesquisa (questionário) junto a especialistas atuantes nas funções da infraestrutura tecnológica para a qualidade. Consubstanciando os propósitos da pesquisa de mestrado, o trabalho explicitou os resultados mais relevantes consolidados pelo amplo e participativo processo de revisão da legislação européia para a livre circulação de produtos e disponibilizou uma metodologia de avaliação. Finalmente, como conclusão, o trabalho definiu os determinantes da revisão do marco regulatório europeu à luz das mudanças de critérios de aplicação das funções da infraestrutura tecnológica.

\section{Palavras-chave}

Metrologia; normalização; avaliação da conformidade; acreditação; fiscalização de mercado; regulação de mercados comuns; livre circulação de produtos. 


\section{Abstract}

Wehmer, Carl Martin; Frota, Mauricio Nogueira (advisor). The role of metrology and metrology-related functions in the regulation of the free movement of goods: considerations on the revision of the European regulatory framework. Rio de Janeiro, 2009. 196 p. MSc. Dissertation - Programa de Pós Graduação em Metrologia. Pontificia Universidade Católica de Rio de Janeiro.

The aim of this M.Sc. dissertation is to analyze the New Regulatory Framework for the free movement of goods in the European market, in the light of the functions of the national quality infrastructure. More specifically, to answer the question posed by the study: will the New (and recently revised) Regulatory Framework be able to mitigate the shortcomings of the previous model? Moreover, the study discusses the significance of metrology, standardization, conformity assessment and accreditation for the effectiveness of market surveillance at a global level and the economic and technical relevance of these processes. The commitment of the Postgraduate Metrology Program (Metrology for Quality and Innovation) to contribute to the improvement of technical regulation, while bridging metrology to citizenship, reflect the motivation of this work. The methodology included: (i) a review of the specialized literature (e.g.: legal documentation and applicable European regulation); (ii) a field research and (iii) the development and application of the structured questionnaire to experts in functions of the quality infrastructure and European legislation. The study highlights the most relevant results of the thorough revision of the European regulation for the free movement of goods. It also provided a methodology of assessment for related issues. In tune with the precepts of the Postgraduate Metrology Programme committed to participate in the debate of current topics in metrology and related matters, the dissertation made available to Brazilian legislators lessons learnt by the European Union on the building of a common market. In conclusion, an experience particularly valuable to those committed to make MERCOSUR a successful undertaking. An endeavor of great proportions where goods, services, capital and labor can circulate freely providing the foundation for prosperity.

\section{Keywords}

Metrology; standardization; conformity assessment; accreditation; market surveillance; regulation of common markets; free movement of goods. 


\section{Sumário}

1. Introdução 14

1.1. Definição do objeto da pesquisa e questões suscitadas 17

1.2. Objetivos da pesquisa de mestrado 19

1.3. Motivação 20

1.4. Metodologia 21

1.5. Estrutura da dissertação 23

2. Regulação de mercados e infraestrutura tecnológica: bases conceituais 25

2.1. Regulação de mercados comuns $\quad 25$

$\begin{array}{ll}\text { 2.1.1. Objetivos da regulação } & 27\end{array}$

2.1.2. Regulação e integração regional 28

2.1.3. O papel das instituições comuns no processo de integração regional 30

2.2. Funções da infraestrutura tecnológica 33

2.2.1. Normalização e Regulamentação Técnica 34

2.2.2. Metrologia $\quad 35$

2.2.3. Avaliação da Conformidade 36

$\begin{array}{ll}\text { 2.2.4. Acreditação } & 37\end{array}$

2.2.5. Fiscalização de mercado 38

2.2.6. Inter-relacionamento entre as funções da infraestrutura tecnológica 39

2.3. O papel da infraestrutura tecnológica na regulação de mercados comuns 41

2.3.1. Atendimento aos preceitos regulatórios $\quad 41$

2.3.2. Participação em mercados comuns 43

3. O marco regulatório europeu para a livre circulação de produtos 45

3.1. A Nova Abordagem e a Abordagem Global - breve histórico 45

3.2. Princípios de funcionamento da Nova Abordagem e Abordagem Global 51

3.2.1. Elementos básicos 52

3.2.2. O papel da normalização 53

3.2.3. O papel da metrologia 56

3.2.4. O papel da avaliação da conformidade e da marcação CE 59

3.2.5. O papel dos organismos notificados 62

3.2.6. O papel da acreditação 63 
4. Estratégia adotada para promover a análise das Abordagens Nova e Global 70

4.1. Disciplina "Regulação e Regulamentação" cursada na PUC-Rio 71

4.2. Curso de capacitação sobre as Abordagens Nova e Global

4.3. Missão técnica brasileira à organizações atuantes na infraestrutura tecnológica da Europa

4.3.1. Objetivos da Missão 73

4.3.2. Composição da delegação brasileira 73

4.3.3. Apresentação das organizações visitadas $\quad 74$

4.3.3.1. Organizações visitadas que atuam na regulamentação técnica $\quad 74$

4.3.3.2. Organizações visitadas que atuam na normalização 77

4.3.3.3. Organizações visitadas que atuam na acreditação 81

4.3.3.4. Organizações visitadas que atuam na avaliação de conformidade $\quad 84$

4.3.3.5. Organizações visitadas que atuam na metrologia 87

4.3.3.6. Organizações visitadas que atuam na fiscalização de mercado 90

4.3.4. Resultados da missão 92

5. A revisão do marco regulatório europeu para a livre circulação de produtos: deficiências detectadas e mudanças introduzidas 93

5.1. Deficiências de maior impacto do marco regulatório europeu vigente 93

5.1.1. Normalização: deficiências detectadas 94

5.1.2. Metrologia: deficiências detectadas 96

5.1.3. Deficiências com respeito à avaliação da conformidade e marcação CE 97

5.1.4. Deficiências com respeito aos organismos notificados 98

5.1.5. Deficiências com respeito à acreditação 100

5.1.6. Deficiências com respeito à fiscalização de mercado 101

5.2. A revisão da Nova e da Abordagem Global: mudanças introduzidas 103

5.2.1. Mudanças introduzidas na normalização 104

5.2.2. Mudanças introduzidas na metrologia 105

5.2.3. Mudanças introduzidas na avaliação da conformidade e marcação CE 105

5.2.4. Mudanças introduzidas na ação dos organismos notificados 106

$\begin{array}{ll}\text { 5.2.5. Mudanças introduzidas na acreditação } & 107\end{array}$

5.2.6. Mudanças introduzidas na fiscalização de mercado 108

$\begin{array}{ll}\text { 5.3. Considerações finais do capítulo } & 109\end{array}$ 
6. Avaliação do marco regulatório europeu para a livre circulação de produtos 111

6.1. Construção da grade analítica da pesquisa 111

6.2. Fundamentos da pesquisa de opinião 112

6.2.1. Construção do questionário 113

$\begin{array}{ll}\text { 6.2.2. Validação do questionário } & 114\end{array}$

6.3. Considerações sobre a pesquisa: população e seleção da amostra 114

6.3.1. Qualificação da amostra utilizada e realização da pesquisa de opinião 115

$\begin{array}{ll}\text { 6.3.2. Significância estatística dos resultados } & 116\end{array}$

6.4. Tabulação de dados, principais resultados e análise dos resultados 117

$\begin{array}{ll}\text { 6.4.1. Normalização } & 119\end{array}$

$\begin{array}{ll}\text { 6.4.2. Metrologia } & 121\end{array}$

6.4.3. Avaliação da conformidade 123

6.4.3.1. O papel dos organismos notificados 123

6.4.3.2. O papel da marcação CE 124

6.4.4. Acreditação 127

6.4.4.1. O papel da acreditação na Nova Abordagem 127

6.4.4.2. O papel da acreditação no novo marco regulatório 129

$\begin{array}{ll}\text { 6.4.5. Fiscalização de mercado } & 131\end{array}$

6.4.5.1. O papel da fiscalização de mercado na Nova Abordagem 131

6.4.5.2. O papel da fiscalização de mercado no novo marco regulatório 133

6.4.6. Discussão sobre as funções da infraestrutura para a qualidade 135

$\begin{array}{ll}\text { 6.4.6.1. Interação entre atores-chave } & 135\end{array}$

$\begin{array}{ll}\text { 6.4.6.2. A eficiência do novo marco regulatório } & 137\end{array}$

$\begin{array}{ll}\text { 6.5. Observações finais } & 138\end{array}$

7. Conclusões e recomendações para trabalhos futuros 142

7.1. O papel da infraestrutura para a qualidade na regulação de mercados

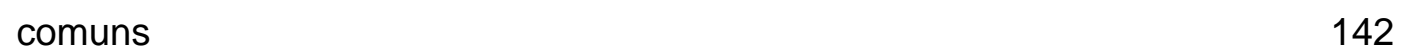

7.2. O marco regulatório europeu: um benchmark atraente 144

7.3. Principais deficiências do atual marco regulatório europeu 145

7.4. Principais mudanças introduzidas pelo novo marco regulatório europeu 146

7.5. Lições aprendidas da experiência européia aplicáveis ao Mercosul 147

7.6. Oportunidades e desafios gerados pelo novo marco regulatório europeu 149

$\begin{array}{ll}\text { 7.7. Uma palavra final } & 151\end{array}$

$\begin{array}{ll}\text { 7.8. Recomendações para trabalhos futuros } & 153\end{array}$ 
8. Referências bibliográficas

155

Abreviaturas e siglas utilizadas

162

Anexo A: Carta de sensibilização

166

Anexo B: Questionário

167

Anexo C: Quadro C1 - Especialistas que aceitaram participar da pesquisa de opinião

Anexo C: Quadro C2 - Tabulação agregada das respostas

de acordo com três categorias de análise

173

Apêndice A: Regulamento CE № 765/2008 do Parlamento Europeu e do Conselho 


\section{Lista de Figuras}

Fig. 1 - Desenho da pesquisa, seus componentes e métodos 22

Fig. 2 - Funções complementares da infraestrutura nacional para a qualidade 40

Fig. 3 - Fluxograma simplificado dos processos de avaliação da conformidade 61

Fig. 4 - Avaliação de risco dos produtos de consumo 68

Fig. 5 - Organizações visitadas na Missão RCT-PME 74

Fig. 6 - Normas ISO por setores 78

Fig. 7 - Secretariados e coordenadores dos TC/SC da IEC em 2007

Fig. 8 - Emissão de CB Test Certificates 85

Fig. 9 - O sistema britânico de medições 89

Fig. 10 - Problemas genéricos de medição encontrados em todos os setores 96 


\section{Lista de Tabelas}

Tab. 1 - Secretariados ISO por países $\quad 78$

Tab. 2 - Acervo normativo CEN/CENELEC 79

Tab. 3 - Resumo normas publicadas ONN (2007) 80

Tab. 4 - Organismos acreditados representados na EA em 2007 e $2008 \quad 82$

Tab. 5 - Resumo organismos nacionais de acreditação 2007

Tab. 6 - Grade analítica da pesquisa 112

\section{Lista de Quadros}

Quadro 1 - Processo de elaboração e adoção de normas européias 